\title{
The Efficacy of a Single Session of 20-Minute Mindful Breathing in Reducing Dyspnea Among Patients With Acute Decompensated Heart Failure: A Randomized Controlled Trial
}

American Journal of Hospice \& Palliative Medicine ${ }^{(}$ I-7

(C) The Author(s) 2020 Article reuse guidelines: sagepub.com/journals-permissions DOI: $10.1177 / 1049909120934743$ journals.sagepub.com/home/ajh

@SAGE

\author{
Diana Leh-Ching Ng, MMED', Chee-Shee Chai, MMED', \\ Kok-Leng Tan, MMED ${ }^{2}$, Kok-Han Chee, MMED ${ }^{3}$, \\ Yu-Zhen Tung, MBBS $^{3}$, Suet-Yen Wai, MBBS $^{3}$, \\ Wei-Ting Joyce Teo, MBBS ${ }^{3}$, Bin-Ting Ang, MBBS ${ }^{3}$, \\ Min-Ai Lim, MBBChBAO ${ }^{3}$, and Seng-Beng Tan, MRCP ${ }^{3}$
}

\begin{abstract}
Heart failure is the leading cause of morbidity and mortality worldwide. Standard treatment for heart failure includes pharmacotherapy and cardiac device implants. However, supportive approaches in managing dyspnea in heart failure are limited. This study aimed to test the efficacy of 20-minute mindful breathing in reducing dyspnea among patients admitted for acute decompensated heart failure. We conducted a parallel-group, non-blinded, randomized controlled trial of a single session of 20-minute mindful breathing plus standard care versus standard care alone among patients admitted for moderate to severe dyspnea due to acute decompensated heart failure, using the dyspnea score based on the Edmonton Symptom Assessment System (ESAS), at the Cardiology Unit of University Malaya Medical Centre in Malaysia. Thirty participants were randomly assigned to a single session of 20-minute mindful breathing plus standard care $(n=15)$ or standard care alone $(n=15)$, with no difference in their demographic and clinical characteristics. There was statistically significant reduction in dyspnea in the intervention group compared to the control group at minute $20\left(\mathrm{U}=49.5, \mathrm{n}_{1}=15, \mathrm{n}_{2}=15\right.$, median reduction in ESAS dyspnea score $1=2$, median reduction in ESAS dyspnea score ${ }_{2}=0$, mean rank $1=11.30$, mean rank $\left.{ }_{2}=19.70, z=-2.692, r=0.4, P=0.007\right)$. Our results provided evidence that a single session of 20-minute mindful breathing was efficacious in reducing dyspnea for patients admitted for acute decompensated heart failure.
\end{abstract}

\section{Keywords}

mindfulness, mindful breathing, dyspnea, heart failure, palliative care

\section{Introduction}

Heart failure is the leading cause of morbidity and mortality worldwide with a prevalence of 26 million. ${ }^{1}$ Comparing to its incidence that has plateaued, the prevalence of heart failure is expected to rise due to the aging population. ${ }^{2}$ Hospitalization for heart failure particularly results in significant socioeconomic burden due to increased healthcare expenditure. ${ }^{2-5}$ It has been estimated that more than half of the total expenditure related to heart failure results from hospitalizations, while pharmacotherapy contributed to $18 \%$ of the cost. ${ }^{4}$

Current standard treatment for congestive heart failure includes pharmacological interventions such as diuretics, $\beta$-blockers, angiotensin-converting enzyme inhibitors, and angiotensin receptor blockers, while nonpharmacological interventions includes patient education for self-care and exercise training to improve functional status. ${ }^{1,6,7}$ There is also usage of device interventions such as implantable cardioverterdefibrillator, cardiac resynchronization therapy, and left ventricular-assisted device. ${ }^{6,7}$ Despite therapeutic advances,

\footnotetext{
'Department of Medicine, Faculty of Medicine and Health Science, University Malaysia Sarawak, Kota Samarahan, Sarawak, Malaysia

${ }^{2}$ Department of Medicine, Faculty of Medicine, University Sains Malaysia, Kelantan, Malaysia

${ }^{3}$ Department of Medicine, Faculty of Medicine, University of Malaya, Kuala Lumpur, Malaysia
}

Corresponding Author:

Seng-Beng Tan, MRCP, Department of Medicine, Faculty of Medicine, University of Malaya, Lembah Pantai, 59100 Kuala Lumpur, Malaysia.

Email: pramudita_I@hotmail.com 